\title{
BOSE-EINSTEIN CONDENSATION AND SUPERFLUIDITY IN TRAPPED ATOMIC GASES
}

\author{
Sandro Stringari \\ Dipartimento di Fisica, Università di Trento, \\ and Istituto Nazionale per la Fisica della Materia, \\ I-38050 Povo, Italy
}

(October 23, 2018)

\begin{abstract}
Bose-Einstein condensates confined in traps exhibit unique features which have been the object of extensive experimental and theoretical studies in the last few years. In this paper I will discuss some issues concerning the behaviour of the order parameter and the dynamic and superfluid effects exhibited by such systems.
\end{abstract}

\section{A. Introduction}

The aim of these lectures is to review some key features exhibited by Bose-Einstein condensation in trapped atomic gases, with special emphasis to the phenomena of superfluidity which have been the object of recent experimental and theoretical investigation and which are characterized in a non trivial way by the combined role of the phase of the order parameter and of two-body interactions.

Let us start recalling that these systems are characterized, at sufficiently low temperatures, by a complex order parameter which can be written as

$$
\Psi_{0}(\mathbf{r}, t)=\sqrt{n_{0}(\mathbf{r}, t)} e^{i S(\mathbf{r}, t)}
$$

where $n_{0}=\left|\Psi_{0}\right|^{2}$ is the so called condensate density and $S$ is the phase. In general the condensate density does not coincide with the density $n$ of the gas, because of the occurrence of quantum and thermal effects. However, in a very dilute gas, where the quantum depletion is negligible and for low temperatures, such that the thermal depletion is small, the condensate density coincides in practice with the total density. For this reason one often identifies $n_{0}$ with $n$. For the same reason in these systems the condensate density can be measured with good precision through imaging techniques. Even at higher temperature the condensate density can be extracted experimentally with reasonable accuracy. In fact, due to the harmonic shape of the external confinement, the thermal component is spacially separated from the condensate so that the density profile is characterized by a typical bimodal structure allowing for systematic experimental analysis. The order parameter of trapped atomic gases then emerges as a crucial quantity not only from the conceptual point of view like in all many-body systems characterized by a broken symmetry, but also from the experimental one. This represents a key difference with respect to superfluid helium where the order parameter is a very hidden variable which can be measured only under very special conditions. The order parameter of a trapped gas can vary in space, time and temperature and characterizes in a direct way most of the physical quantities that one measures in these systems.

In the first part I will mainly discuss the properties of the modulus of the order parameter. From the theoretical point of view this quantity is interesting because even its shape at equilibrium is very sensitive to the presence of two-body interactions. In the second part I will give more emphasis on the role of the phase, a quantity which plays a crucial role in characterizing the coherence and superfluid features of our systems. Of course the modulus and the phase of the order parameter are deeply related quantities and in many cases their behaviour cannot be discussed in a separate way. Many of the results discussed in these notes are derived in more detail in ref. 1] to which we refer also for a more complete list of references.

The discussion on Bose-Einstein condensation presented in these notes is stimulated by several features of fundamental interest that are worth mentioning. In particular it is important to recall that

- BEC gases in traps provide a unique opportunity to study the transition from the microscopic to the macroscpic world.

- In these systems BEC shows up both in momentum and coordinate space.

- BEC gases provide an almost ideal realization of classical matter waves, giving rise to new coherence phenomena.

- Trapped gases are well suited to study rotational superfluid phenomena. 


\section{B. BEC is a phase transition}

In the presence of harmonic trapping

$$
V_{\text {ext }}=\frac{1}{2} m\left(\omega_{x}^{2} x^{2}+\omega_{y}^{2} y^{2}+\omega_{z}^{2} z^{2}\right)
$$

the critical temperature for Bose-Einstein condensation of an ideal gas can be easily evaluated using the standard techniques of quantum statistical mechanics. To this purpose one has to define the thermodynamic limit. In the case of harmonic trapping the natural choice is to take $N \rightarrow \infty$ and $\omega_{h o} \rightarrow 0$, with the combination $N \omega_{h o}^{3}$ kept fixed. Here $\omega_{h o}=\left(\omega_{x} \omega_{y} \omega_{z}\right)^{1 / 3}$ is the geometrical average of the three harmonic frequencies. Notice that in this limit the system, differently from traditional thermodynamic bodies, is characterized by a non uniform density varying on a macroscopic scale, much larger than the typical interatomic distance. The critical temperature takes the value

$$
k_{B} T_{c}=0.94 \hbar \omega_{h o} N^{1 / 3}
$$

while the condensate fraction obeys the law

$$
\frac{N_{0}}{N}=1-\left(\frac{T}{T_{c}}\right)^{3}
$$

for $T<T_{c}$. Similarly to the case of the uniform gas one predicts a phase transition even in the absence of two-body interactions. This unique prediction of quantum statistical mechanics can be now tested experimentally in trapped Bose gases [2,3]. In fact, close to the critical temperature, these gases are so dilute that one can neglect in first approximation the role of the interaction. Estimate (3) has actually guided experimentalists to the right domain of temperatures which turns out to be of the order of $\mu K$ (typically the trapping frequencies are of the order of a few $n K$ while the number $N$ of atoms varies from $10^{4}$ to $10^{7}$ ). Also the temperature dependence of the condensate fraction turns out to be in reasonable agreement with the theoretical prediction (4) as shown by fig 1.

An interesting question is how two-body forces modify the ideal gas estimate (3). Interaction effects can have two different origins. A first one is a genuine many-body effect exhibited by uniform systems where one works at fixed density. This effect is highly non trivial from the theoretical point of view [4] but the resulting correction is expected to be small and difficult to observe in trapped gases. A second effect is the result of mean field forces which, in the case of positive scattering lengths, tend to push the gas towards the external region, thereby reducing the density of the gas. This causes a natural decrease of the critical temperature. The opposite happens if the interaction is attractive. The mean field effect can be easily calculated and the result takes the form [5]

$$
\frac{\delta T_{c}}{T_{c}}=-1.3 \frac{a}{a_{h o}} N^{1 / 6}
$$

where $a$ is the scattering length and $a_{h o}=\sqrt{\hbar / m \omega_{h o}}$ is the oscillator length. Typical values for $\delta T_{c} / T_{c}$ are a few percent in the available traps and the present accuracy of experimental data does not allow for a quantitative check of this prediction. By tuning the value of the scattering length to larger values it should be possible to obtain more sizable corrections to the value of the critical temperature.

The above discussion has introduced a first important combination of the relevant parameters of the problem, given by $N^{1 / 6} a / a_{h o}$. This combination fixes the importance of interaction effects on the thermodynamic properties of the system. Its origin is easily understood if one calculates the ratio between the interaction energy $E_{\text {int }} \sim g n$ where $g=4 \pi \hbar^{2} a / m$ is the coupling constant fixed by the scattering length and $n$ is the density of the thermal cloud evaluated in the center of the trap, and the thermal energy $E_{T} \sim k_{B} T$. A simple estimate for the thermal density is given by the classical value $n \sim N\left(k_{B} T / m \omega_{h o}\right)^{3 / 2}$. Using result (3) for the critical temperature, one finds that the ratio $E_{\text {int }} / E_{T}$, evaluated at $T \sim T_{c}$, is actually fixed by the combination $N^{1 / 6} a / a_{h o}$. This should not be confused with the so called Thomas-Fermi combination $N a / a_{h o}$ which instead characterizes the effects of two body-interactions on the zero temperature properties of a trapped condensate. The Thomas-Fermi combination is recovered by evaluating the ratio between the interaction energy $E_{i n t} \sim g n$, where $n$ is now the central value of the condensate density, and the quantum oscillator energy $\hbar \omega_{0}$. The dependence on $N$ given by the two combinations discussed above is extremely different and explains why the effects of the interaction at $T \sim T_{c}$ are small, while at $T \sim 0$ they are so large that cannot be treated in a perturbative way. The role of the Thomas-Fermi parameter will be discussed systematically in the next sections.

Before concluding this section it is useful to remind that the measurements of the temperature dependence of the condensate density available in trapped Bose gases are more reliable than the ones obtained in superfluid helium [6] 
(see fig.2). In helium the condensate fraction is not a natural observable and can be extracted only through elaborated analysis of neutron scattering data at high momentum transfer. Actually the main evidence for the phase transition in superfluid helium comes from the analysis of other thermodynamic quantities, like the viscosity and the specific heat.

\section{Order parameter and long range order}

A key quantity characterizing the long range order of a many-body system is the one-body density matrix [7]:

$$
n\left(\mathbf{r}, \mathbf{r}^{\prime}\right)=\left\langle\hat{\Psi}^{\dagger}(\mathbf{r}) \hat{\Psi}\left(\mathbf{r}^{\prime}\right)\right\rangle
$$

where $\hat{\Psi}(\mathbf{r})=\hat{a}_{0} \phi_{0}(\mathbf{r})+\sum_{i \neq 0} \hat{a}_{i} \phi_{i}(\mathbf{r})$ is the field operator expressed in terms of the annihilation operators relative to a generic basis of single particle wave functions. We have here separated the contribution arising from the lowest single particle state $\phi_{0}$ in order to emphasize the effect of BEC.

Let us first consider an ideal gas at zero temperature. In this case all the particles occupy the same state $\phi_{0}$ determined by the solution of the Schrödinger equation for the single particle Hamiltonian $-\hbar^{2} \nabla^{2} / 2 m+V_{e x t}$. The density matrix (6) then takes the separable form $n^{(1)}\left(\mathbf{r}, \mathbf{r}^{\prime}\right)=N \phi_{0}^{*}(\mathbf{r}) \phi_{0}\left(\mathbf{r}^{\prime}\right)$ and remains different from zero for macroscopic distances $\left|\mathbf{r}-\mathbf{r}^{\prime}\right|$ of the order of the size of the sample (long range order). This is also called first order coherence and is a key consequence of Bose statistics.

At finite temperatures one should include the thermal occupation of the other single particle states and eq.(6) yields

$$
n\left(\mathbf{r}, \mathbf{r}^{\prime}\right)=N_{0} \phi_{0}^{*}(\mathbf{r}) \phi_{0}\left(\mathbf{r}^{\prime}\right)+\sum_{i \neq 0} n_{i} \phi_{i}^{*}(\mathbf{r}) \phi_{i}\left(\mathbf{r}^{\prime}\right)
$$

where $n_{i}=\left(\exp \beta\left(\epsilon_{i}-\mu\right)-1\right)^{-1}$ are the thermal occupation numbers and $\epsilon_{i}$ are the single-particle excitation energies of the excited states $\phi_{i}$. In the thermodynamic limit the ratio $N_{0} / N$ tends to a constant value (condensate fraction) giving rise to long range order, while the second term in the r.h.s. of eq. (7) can be replaced with an integral and consequently vanishes at large distances. The importance of long range order is hence reduced at finite temperature since $N_{0}$ is smaller than at $T=0$. For a uniform ideal gas the decay of the thermal component is given by $n(s) \rightarrow k T m /(4 \pi s)$ where $s=\left|\mathbf{r}-\mathbf{r}^{\prime}\right|$. Above the critical temperature one has no more long range order $\left(N_{0} / N \rightarrow 0\right)$ and the one-body density matrix decays faster than $1 / s$ at large distances. In the limit $T \gg T_{c}$ one finds the gaussian behaviour $n(s)=n(0) \exp \left[-s^{2} m k_{B} T / 2 \hbar^{2}\right]$.

The effects due to Bose-Einstein condensation discussed above can be also derived by setting $\hat{a}_{0}=\hat{a}_{0}^{\dagger} \equiv \sqrt{N_{0}}$. This is the well known Bogoliubov prescription which corresponds to ignoring the non commutativity between the particle operators $a_{0}$ and $a_{0}^{\dagger}$ and treating them like $c$-numbers. In virtue of the Bogoliubov assumption the field operator takes the form

$$
\hat{\Psi}(\mathbf{r})=\Psi_{0}(\mathbf{r})+\sum_{i \neq 0} \hat{a}_{i} \phi_{i}(\mathbf{r})
$$

where

$$
\Psi_{0}(\mathbf{r})=\sqrt{N_{0}} \phi_{0}(\mathbf{r})
$$

is a classical field, called the order parameter. The order parameter can be also regarded as the expectation value of the field operator

$$
\Psi_{0}(\mathbf{r})=\langle\hat{\Psi}(\mathbf{r})\rangle
$$

where the average is taken on a configuration with broken gauge symmetry. In terms of the order parameter the diagonal term of the density matrix can be written as

$$
n(\mathbf{r})=n_{0}(\mathbf{r})+n_{T}(\mathbf{r})
$$

where $n_{0}(\mathbf{r})=\left|\Psi_{0}(\mathbf{r})\right|^{2}$ is the condensate density and $n_{T}(\mathbf{r})=\sum_{i \neq 0} n_{i}\left|\phi_{i}(\mathbf{r})\right|^{2}$ is the contribution arising from the particles out of the condensate. In a dilute gas $n_{T}$ can be safely identified with the density of the thermal component. In general one should not however ignore the fact that even at zero temperature the total density is different from the condensate density since the occupation numbers $n_{i}$ with $i \neq 0$ of eq.(17) differ from zero, giving rise to the quantum 
depletion of the condensate. An example where quantum depletion plays a crucial role is presented in fig. 3 which reports the density profile of a cluster of helium atoms calculated at $T=0$ using a correlated basis approach [8]. These systems, differently from atomic gases, are strongly correlated. The figure clearly shows that the contribution of the condensate is only a small fraction (about 10\%) and that in this case the measurement of the density profile would not yield any useful information on the condensate density $n_{0}$ even at zero temperature. In the calculation of ref. [8] the condensate density has been evaluated by determining the natural orbits which diagonalize the 1-body density matrix (see eq.(7))

\section{Equation for the order parameter}

The equation for the order parameter can be derived starting from the Heisenberg equation for the field operator

$$
\begin{aligned}
i \hbar \frac{\partial}{\partial t} \hat{\Psi}(\mathbf{r}, t)=[\hat{\Psi}(\mathbf{r}, t), \hat{H}]=\left[-\frac{\hbar^{2} \nabla^{2}}{2 m}+V_{e x t}(\mathbf{r}, t)\right. \\
\\
\left.+\int \hat{\Psi}^{\dagger}\left(\mathbf{r}^{\prime}, t\right) V\left(\mathbf{r}^{\prime}-\mathbf{r}\right) \hat{\Psi}\left(\mathbf{r}^{\prime}, t\right) d \mathbf{r}^{\prime}\right] \hat{\Psi}(\mathbf{r}, t) .
\end{aligned}
$$

This is an exact equation if one uses for $V(s)$ the exact two-body interaction between particles and holds not only for dilute gases, but also for strongly interacting systems like superfluid ${ }^{4} \mathrm{He}$. A closed equation for the order parameter is obtained if we replace the field operator $\Psi$ with the classical field $\Psi_{0}$ or, equivalently, with its expectation value (10). This procedure is correct provided a series of assumptions are satisfied:

i) The thermal depletion should be small so that the thermal fluctuations of the field operator can be ignored. This implies temperatures much smaller than the critical value.

ii) The system should be so dilute that the quantum fluctuations of the field operator can be ignored. This requires that the gas parameter $n a^{3}$ be much smaller than unity or, equivalently, that the average distance between particles be much larger than the scattering length.

iii) The microscopic interaction $V(s)$ should be replaced by the pseudopotential $g 4 \pi \hbar^{2}(a / m) \delta(\mathbf{s})$ fixed by the $s$-wave scattering length $a$. This implies that only low energy features of the problem can be investigated with this approach. Equivalently, only variations of the order parameter over distances larger than the range of the interaction can be explored.

iv) The number of atoms $N$ should be large enough in order to justify the Bogoliubov prescription.

Under the hypothesis i)-iv) one obtains the most famous Gross-Pitaevskii equation [9]

$$
i \hbar \frac{\partial}{\partial t} \Psi_{0}(\mathbf{r}, t)=\left(-\frac{\hbar^{2} \nabla^{2}}{2 m}+V_{e x t}(\mathbf{r}, t)+g\left|\Psi_{0}(\mathbf{r}, t)\right|^{2}\right) \Psi_{0}(\mathbf{r}, t),
$$

for the classical field $\Psi_{0}$. This equation provides the basic tool to explore the static and dynamic features of non uniform Bose gases at low temperature. The GP equation shares interesting analogies with the Maxwell equations of classical electromagnetism. In both approaches one provides a description of a many-body problem (atoms anf photons respectively) in terms of a classical field (order parameter and electromagnetic field ). From this point of view a gas of Bose-Einstein condensed atoms can be regarded as a classical matter wave. With respect to the equations of electromagnetism the GP equation however exhibits important differences: first it contains an important non linear term due to interactions (this difference is not crucial since non lineariteies are exhibited also by electromagnetic phenomena in dispersive media); a second important difference is the role played by the Planck constant $\hbar$. Differently from the Maxwell equations the GP equation (13) in fact depends explicitly on this fundamental constant of quantum mechanics. This feature is directly connected with the different momentum-energy relationship exhibited in the two cases. For massive particles the relation is quadratic $\left(E=p^{2} / 2 m\right)$, while for photons it is linear $(E=c p)$. When we employ the ondulatory description through the de Broglie prescription $E=\hbar \omega$ and $p=\hbar k$, the relationship bewteen the wavevector and the frequency is still independent on the Planck constant in the case case of photons $(\omega=c k)$, while it exhibits an explict dependence on $\hbar$ in the case of atoms $\left(\omega=\hbar k^{2} / 2 m\right)$. This explains, for example, why interference phenomena with classical matter waves depend explicitly on the value of the Planck constant.

\section{E. Ground state configuration}

If we look for stationary solutions of the form 


$$
\Psi_{0}(\mathbf{r}, t)=\Psi_{0}(\mathbf{r}) \exp (-i \mu t / \hbar)
$$

the Gross-Pitaevskii equation (13) becomes

$$
\left(-\frac{\hbar^{2} \nabla^{2}}{2 m}+V_{e x t}(\mathbf{r})-\mu+g\left|\Psi_{0}(\mathbf{r})\right|^{2}\right) \Psi_{0}(\mathbf{r})=0 .
$$

This equation can be also derived using the standard variational procedure

$$
\delta(E-\mu N)=0
$$

where

$$
E=\int\left(\frac{\hbar^{2}}{2 m}\left|\nabla \Psi_{0}\right|^{2}+V_{\text {ext }}(\mathbf{r})\left|\Psi_{0}\right|^{2}+\frac{g}{2}\left|\Psi_{0}\right|^{4}\right) d \mathbf{r}=E_{k i n}+E_{\text {ext }}+E_{\text {int }}
$$

is the energy functional of the system. In this equation we have naturally identify the kinetic energy $\left(E_{k i n}\right)$, the external potential $\left(E_{\text {ext }}\right)$ and the mean field interaction $\left(E_{\text {int }}\right)$ energies. Starting from the GP equation one can easily derive the useful relationship

$$
N \mu=E_{\text {kin }}+E_{\text {ext }}+2 E_{\text {int }}
$$

for the chemical potential, and the virial identity

$$
2 E_{\text {kin }}-2 E_{\text {ext }}+3 E_{\text {int }}=0
$$

characterizing the equilibrium configuration. It is also useful to recall the so called release energy

$$
E_{\text {release }}=E_{\text {kin }}+E_{\text {int }}
$$

which coincides with the energy of the system after switching off the trap and is hence given by the sum of the kinetic and interaction energies.

It is important to remind here that the quantity $E_{k i n}$ entering the above equations is the kinetic energy of the condensate (mean field kinetic energy) and should not be confused with the full kinetic energy of the system. The latter takes in fact contribution also from the particles out of the condensate and, using the notation (7) for the 1-body density matrix, can be written in the form

$$
E_{\text {kin }}^{\text {total }}=\int d \mathbf{r} \frac{\hbar^{2}}{2 m}\left(\left|\nabla \Psi_{0}(\mathbf{r})\right|^{2}+\sum_{i \neq 0} n_{i}\left|\nabla \phi_{i}(\mathbf{r})\right|^{2}\right) .
$$

While for dilute gases the contribution $\sum_{i \neq 0} n_{i}$ of the non condensate component to the total number of atoms is small at $T=0$ and one can safely identify $N$ with $N_{0}$, the corresponding contribution to the kinetic energy (second term of eq.(21)) may be much larger than the condensate kinetic energy. For similar reasons the mean field interaction energy $E_{\text {int }}$ should not be confused with the full interaction energy whose evaluation requires the explicit knowledge of short range correlations. The distinction between the mean field and total energies becomes particularly clear and stark in the case of a uniform gas interacting with hard sphere potentials. In this case the total kinetic energy coincides with the energy of the system, the interaction energy being exactly zero due to the special choice of the potential. Conversely the kinetic energy of the condensate is zero because of the homogeneity of the sample. Notice that the mean field scheme fails to evaluate the full kinetic energy (21). This can be seen in the case of a uniform gas where the occupation number of the single particle states is given by the Bogoliubov expression $n_{p}=\left(p^{2} / 2 m+m c^{2}\right) / 2 \epsilon(p)-1 / 2$. The quantity $\sum_{p \neq 0} n_{p}$ is evaluable and gives the quantum depletion of the condensate. Viceversa the sum $\sum_{i \neq 0}\left(p^{2} / 2 m\right) n_{p}$ exhibits an ultraviolet divergency, showing that the evaluation of the kinetic energy requires the proper knowledge of the momentum distribution at momenta of the order of the inverse of the scattering length and cannot be achieved within the mean field scheme.

It is finally worth pointing out the key role played by the chemical potential in the formalism of the GP equation. As one can see from (14) the time dependence of the order parameter is fixed by $\mu$ and not by the energy. This is a consequence of the fact that the order parameter is not a wave function and that the GP equation is not a Schrödinger equation in the usual sense of quantum mechanics. From the point of view of many-body theory the order parameter corresponds to the matrix element of the field operator between two many-body wave functions containing, 
respectively, $N$ and $N+1$ particles. This implies that its time dependence is fixed by the factor $e^{-i(E(N+1)-E(N)) t}$ and hence by the chemical potential $\mu=\partial E / \partial N$ rather than by the energy $E$.

If the Thomas Fermi parameter $N a / a_{h o}$ is very large the GP equation (13) can be solved analytically. In fact in this case the density profile, as a consequence of the repulsive effect of the interactions, becomes so smooth that the kinetic energy term in (13) can be ignored and the density takes the shape of an inverted parabola (we consider here spherical trapping)

$$
n(\mathbf{r})=\frac{1}{g}\left(\mu-V_{e x t}\right)=\frac{\mu}{g}\left(1-\frac{r^{2}}{R^{2}}\right)
$$

where the radius $R$ of the condensate is given by the formula

$$
R=a_{h o}\left(15 N \frac{a}{a_{h o}}\right)^{1 / 5}
$$

and $\mu=m \omega_{h o}^{2} R^{2} / 2$. For an axially deformed trap the density profile is simply generalized to $n(\mathbf{r})=(\mu / g)\left(1-r_{\perp}^{2} / R_{\perp}^{2}-\right.$ $\left.z^{2} / Z^{2}\right)$ where $r_{\perp}^{2}=x^{2}+y^{2}$ and the radial and axial radii are related by the equation $m \omega_{\perp}^{2} R_{\perp}^{2}=m \omega_{z}^{2} Z^{2}=2 \mu$. In many available configurations the size of the atomic cloud can become much larger than the oscillator length and reach almost macroscopic values.

\section{F. Visibility of the condensate}

In the introduction we have emphasized the important fact that, due to the harmonic trapping, the condensate density can be separated from the thermal component, a crucial condition for the experimental visibility of the condensate. Due to the importance of this fact it is worth discussing in a deeper way the consequences of two-body interactions. In fact the argument usually invoqued is based on the ideal gas model where the width of the condensate is fixed by the oscillator length $a_{h o}=\sqrt{\hbar / m \omega_{h o}}$. On the other hand the width of the thermal cloud is of the order of

$$
R_{T} \sim\left(\frac{k_{B} T}{m \omega_{h o}^{2}}\right)^{1 / 2}
$$

so that, for "macroscopic" temperatures $k_{B} T \gg \hbar \omega_{h o}$, one has $R_{T} \gg a_{h o}$ with the consequent net separability of the two components. In the previous section we have however shown that interactions are responsible for a huge increase of the size of the condensate whose value, in the Thomas-Fermi limit, is given by eq.(23). One then conclude that interacations will partially reduce the visibility of the condensate by making its size comparable to the one of the thermal cloud. Interactions, by producing a smoother density profile, make the system closer to a uniform gas and consequently reduce the visibility of BEC in coordinate space. In fig 4 we show a typical in situ measurement of the density profile taken at different temperatures [10]. A similar argument holds if one explores the behaviour of the release energy $E_{\text {release }}$. For an ideal gas the release energy is fixed by the oscillator energy $\hbar \omega_{0}$, which is much smaller than the release energy of the thermal cloud, fixed by $k_{B} T$. This provides a net separation between the two components which expand at different velocities. The difference is sizably reduced if one takes into account interactions which increase significantly the value of $E_{\text {release }}$ making it comparable to the thermal energy $k T$. In conclusion the separability of the condensate from the thermal component is significantly reduced by two-body interactions and requires a careful bimodal fit to the measured profiles (both in in situ and after expansion).

While the visibility of the condensate in coordinate space is reduced by two-body (repulsive) interactions, it is conversely enhanced in momentum space. In fact, according to the Heisenberg relationship, the width of the condensate in momentum space behaves like $\hbar / R$ and hence becomes smaller and smaller as $R$ increases, approaching the typical $\delta$ distribution characterizing BEC in uniform systems. While in the absence of interactions the visibility of BEC in coordinate and in momentum space are perfectly equivalent, due to the symmetric role played by the spatial and momentum coordinates of the harmonic Hamiltonian, in the Thomas-Fermi limit this symmetry is lost. The possibility of measuring the momentum distribution of trapped atomic clouds, via inelastic photon scattering experiments $[11]$ opens new possibilities to separate at a deeper level the condensate from the thermal component. In principle such measurements might provide an identification of the quantum depleted part too. In practice the effect of quantum depletion is too small to be observed in the presently available condensates. 


\section{G. Role of the phase: interference with BEC}

The phase of the order parameter plays a crucial role in characterizing the interference phenomena exhibited by a Bose-Einstein condensed gas. The simplest example is the interference produced by two initially separated condensates which expand and overlap. The corresponding experiment was first carried out at Mit [12].

Let us first consider a single expanding condensate. At large times the phase of the order parameter takes a quadratic dependence, yielding the asymptotic form

$$
\Psi_{0}(\mathbf{r}, t)=\left|\Psi_{0}(\mathbf{r}, t)\right| e^{i m r^{2} / 2 \hbar t}
$$

for the order parameter. It is interesting to notice that the velocity field

$$
\mathbf{v}=\frac{\hbar}{m} \nabla S
$$

associated with the order parameter (25) coincides with the classical law $\mathbf{v}=\mathbf{r} / t$. For large times the velocity then exhibits a fully classical behaviour. However the phase $S$ keeps its quantum nature fixed by the Planck constant.

If we now consider two expanding condensates separated by a distance $\mathbf{d}$ and we assume that for large times the order parameter is given by a linear combination of the form

$$
\Psi_{0}=\left|\Psi_{1}\right| e^{i m(\mathbf{r}+\mathbf{d} / 2)^{2} /(2 \hbar t)}+e^{i \alpha}\left|\Psi_{2}\right| e^{i m(\mathbf{r}-\mathbf{d} / 2)^{2} /(2 \hbar t)}
$$

we find that the density $\left|\Psi_{0}\right|^{2}$ acquires a modulation with typical fringes orthogonal to the vector $\mathbf{d}$ and separated by the distance

$$
\lambda=\frac{h t}{m d}
$$

This distance depends explictly on the Planck constant and with typical choices of the parameters $d$ and $t$ is a visibile length of the order of $10-20 \mu \mathrm{K}$. The above estimate for the interference effects ignores the role of the interaction between the two clouds which are responsible, during the expansion, for deviations from the law (27). The basic physics is however accounted by this simple model which provides a useful qualitative estimate of interference effects. It points out, in particular, a conceptually important question connected with the role of the relative phase of the two condensates. Suppose that the two condensates are built in an independent way before measurement. Why should the order parameter have the form (27) with a well defined value of the relative phase $\alpha$ giving rise to interference? The question addresses the important problem of the quantum measurement and of the consequent reduction of the wave packet. The standard theoretical point view, based on the traditional rules of quantum mechanics, predicts that after measurement the wave function of the whole system will be of the form (27) with a relative phase which cannot predicted in advance, unless the condensates were already in a coherent configuration before the expansion.

\section{H. Role of the phase: irrotationality of the superfluid flow}

A crucial consequence of the existence of an order parameter of the form (1), is the irrotationality of the flow. This feature is expressed by eq.(26) from which one deduces that the phase plays the role of a velocity potential. Actually starting from the Gross-Pitaevskii equation for the order parameter it is possible to derive the equations of motion in a form which resembles the equations of irrotational hydrodynamics. These are the basic equations for the describing the motion of a superfluid. The derivation is straightforward in a dilute gas. One starts from expression (1) for the order parameter and derives, using the GP equation $(13)$, a closed set of equations for the density and for the phase or, equivalently, for the velocity field. The equations take the form [13]

$$
\begin{gathered}
\frac{\partial n}{\partial t}+\operatorname{div}(\mathbf{v} n)=0 \\
\hbar \frac{\partial}{\partial t} S+\left(\frac{1}{2} m \mathbf{v}^{2}+V_{e x t}+g n-\frac{\hbar^{2}}{2 m \sqrt{n}} \nabla^{2} \sqrt{n}\right)=0 .
\end{gathered}
$$

The first equation is the usual equation of continuity, while the second one can be regarded as an equation for the velocity potential. Notice that in these equations, which are exactly equivalent to the original Gross-Pitaevskii 
equation, the Planck constant enters through the so called quantum kinetic pressure term proportional to $\nabla^{2} \sqrt{n}$. This term should be compared with the interaction $g n$ given by two-body forces. If the role of interactions is very important the quantum pressure term can be neglected. This happens in the study of the equilibrium profile in the Thomas-Fermi limit. The quantum pressure term is negligible also in the time dependent problem, provided we study phenomena where the density varies in space on lengths scales $L$ larger than the so called healing length

$$
\xi=\sqrt{\frac{\hbar}{2 m g n}} .
$$

If $L \gg \xi$ it is immediate to see that the quantum pressure in (30) is negligible and that the same equation reduces to the simpler Euler-like form

$$
m \frac{\partial}{\partial t} \mathbf{v}+\nabla\left(\frac{1}{2} m \mathbf{v}^{2}+V_{e x t}+\mu\right)=0
$$

where $\mu=g n$ is the chemical potential evaluated for a uniform body at the corresponding density. The equation of continuity and the Euler equation (32) are called the hydrodynamic equations of superfluids. They are characterized by two important features: the irrotationality of the motion and the crucial role of interactions which suppress the effects of the quantum pressure. It is worth noticing that the "hydrodyinamic" form of these equations is not the result of collisional processes as happens in classical gases, but is the consequence of superfluidity.

It is also useful to recall that the validity of the hydrodynamic equations is limited to the low $T$ regime (at finite temperature one should use the formalism of two-fluid hydrodynamics). In the limit of small temperatures these equations can be used to study also dense superfluids, like ${ }^{4} \mathrm{He}$ as well as Fermi superfluids. Of course in this case one should use the corresponding expression for the chemical potential. It is finally worth mentioning that in the hydrodynamic limit the Planck constant, which entered equation (30) for the velocity field through the quantum pressure term, has disappeared from the equations of motion.

Starting from the hydrodynamic equations of superfluids one can explore different interesting problems in trapped BEC gases. These include the propagation of sound and the excitation of collective modes.

\section{Sound and collective excitations}

In the limit of small perturbations (linear limit) the hydrodynamic equations (29) and (32) can be reduced to the typical form

$$
\frac{\partial^{2}}{\partial t^{2}} \delta n=\nabla \cdot\left[c^{2}(\mathbf{r}) \nabla \delta n\right]
$$

characterizing the propagation of sound waves in nonuniform media. Here $c(\mathbf{r})$ is a $\mathbf{r}$-dependent sound velocity fixed by the relationship $m c^{2}(\mathbf{r})=\mu-V_{e x t}(\mathbf{r})$. Sound can propagate in trapped Bose gases if the wavelength $\lambda$ of the wave is smaller than the size of the system. In the Thomas-Fermi limit this condition can be well satisfied especially in the elongated direction of the condensate. In the experiments carried out at MIT [14] the axial size $Z$ is a few hundred micrometers while $\xi$ is a fraction of micrometer, so there is wide space for the propagation of sound waves in such samples. It is also interesting to consider the case where the wave length $\lambda$ is smaller than the axial size $(\lambda<Z)$, but larger than the radial size $\left(\lambda>R_{\perp}\right)$. In this case the nature of the wave is characterized by typical 1-dimensional features 15 .

If the wavelength becomes comparable to the size of the condensate the solutions of eq.(33) cannot be described in terms of a localized propagation of sound, but should be determined globally. These solutions correspond to oscillations of the whole system and the discretization of the eigenvalues cannot be ignored in this case. For spherical trapping one can obtain analytic solutions of (33) in the form $\delta n=R_{n_{r} \ell} Y_{\ell m}$ where $\ell=0,1,2 .$. is the angular momentum (in units of $\hbar$ ) carried by the excitation, $n_{r}$ is the number of the radial nodes and $R_{n \ell}$ is the radial function to be determined by solving the equations of hydrodynamics. The corresponding eigenfrequencies obey the dispersion law 13

$$
\omega_{H D}=\omega_{h o} \sqrt{2 n_{r}^{2}+2 n_{r} \ell+3 n_{r}+\ell}
$$

which should be compared with the prediction $\omega_{h o}\left(2 n_{r}+\ell\right)$ of the ideal gas model. The role of interactions is particularly evident for the surface excitations $\left(n_{r}=0\right)$. In this case the hydrodynamic theory gives $\sqrt{\ell} \omega_{h o}$ to be compared with value $\ell \omega_{0}$ of the ideal gas prediction. 
Result (34) reveals that, in the Thomas-Fermi limit $N a / a_{\mathrm{ho}} \gg 1$, the dispersion relation of the normal modes of the condensate has changed significantly from the noninteracting behavior, as a consequence of two-body interactions. However it might appear surprising that in this limit the dispersion does not depend any more on the value of the interaction parameter $a$. This differs from the uniform case where the dispersion law, in the corresponding phonon regime, is given by $\omega=c q$ and depends explicitly on the interaction through the velocity of sound. The behavior exibited in the harmonic trap is well understood if one notes that the discretized values of $q$ are fixed by the boundary and vary as $1 / R$ where $R$ is the size of the system. While in the box this size is fixed, in the case of harmonic confinement it increases with $N$ due to the repulsive effect of two-body interactions (see eq.(23): $R \sim\left(N a / a_{\mathrm{ho}}\right)^{2 / 5}\left(m \omega_{\mathrm{ho}}\right)^{-1 / 2}$. On the other hand the value of the sound velocity, calculated at the center of the trap, is given by $c=\left(N a / a_{\mathrm{ho}}\right)^{2 / 5}\left(\omega_{\mathrm{ho}} / m\right)^{1 / 2}$ and also increases with $N$. One finally finds that in the product $c q$ both the interaction parameter and the number of atoms cancel out, so that the frequency turns out to be proportional to the bare oscillator frequency $\omega_{\text {ho }}$. A similar argument holds also for the surface excitations. In fact in the presence of an external force the dispersion relation of the surface modes obeys the classical law $\omega^{2}=F q / m$ where $F=m \omega_{h o}^{2} R$ is the force evaluated at the surface of the system. Since the product $\hbar q R$ gives the angular momentum carried by the surface wave, one immediately recovers the dispersion law $\sqrt{\ell} \omega_{h o}$.

Analytic solutions of the HD equation (33) are available also in the case of axi-symmetric potentials of the form $V_{\text {ext }}=(m / 2)\left(\omega_{\perp}^{2} r_{\perp}^{2}+\omega_{z}^{2} z^{2}\right)$. In this case the third component of angular momentum is still a good quantum number. For very elongated traps $\left(\omega_{z} \ll \omega_{\perp}\right)$ the frequency of the lowest solution of even parity takes the value [13] $\sqrt{5 / 2} \omega_{z}$ in excellent agreement with the experimental results of [16].

The high precision of frequency measurements is not only providing us with a powerful tool to check the predictions of the hydrodynamic theory of superfluids, but can be also used to explore finer effects, like the temperature dependence of the collective frequencies and, possibly, beyond mean field corrections.

\section{J. Moment of inertia}

An important consequence of the hydrodynamic theory of superfluids is that the response

$$
\Theta=\lim _{\Omega \rightarrow 0} \frac{<L_{z}>}{\Omega}
$$

to a rotating field of the form $H_{\text {rot }}=-\Omega L_{z}$, where $L_{z}=-i \hbar \sum_{k} \mathbf{r}_{k} \times \nabla_{k}$ is the third component of the angular momentum operator, is given by the irrotational value

$$
\Theta=\left(\frac{<x^{2}-y^{2}>}{<x^{2}+y^{2}>}\right)^{2} \Theta_{\text {rig }}
$$

of the moment of inertia where $\Theta_{\text {rig }}$ is the classical rigid value. This result can be easily derived by rewriting the equations of hydrodynamics in the frame rotating with the angular velocity $\Omega$. Eq.(36) can be also written in the form $\Theta=\epsilon^{2} \Theta_{i r r}$ where $\left.\epsilon=\left(\omega_{x}^{2}-\omega_{y}^{2}\right) / \omega_{x}^{2}+\omega_{y}^{2}\right)$ is the deformation of the trap in the plane of rotation and we have used the Thomas-Fermi results for $\left\langle x^{2}\right\rangle$ and $\left\langle y^{2}\right\rangle$.

The quenching of the moment of inertia with respect to the rigid value was confirmed in a series of experiments of the 60's caried out on superfluid helium, providing an independent measurement of the superfluid density [17]. In this context it is worth mentioning that the superfluid density of superfluid helium exhibits a very different behaviour with respect to the condensate density, especially at low temperature. In particular at $T=0$ the superfluid density coincides with the total density while the condensate density is only a small fraction $(\sim 10 \%)$.

A challenging question is how to measure the moment of inertia of a trapped gas where the direct measurement of the angular momentum $L_{z}$ is not feasible due to the diluteness of the sample. An interesting possibility is provided by the fact that, if the deformation of the trap is different from zero, the quadrupole and rotational degrees of freedom are coupled each other. This is well understood by considering the exact commutation relation

$$
\left[H, L_{z}\right]=i m\left(\omega_{x}^{2}-\omega_{y}^{2}\right) Q
$$

which explicitly points out the link between the angular momentum operator $L_{z}$ and the quadrupole operator $Q=$ $\sum_{i} x_{i} y_{i}$. Since the quadrupole variable can be easily excited and imaged one expects to obtain information on the rotational properties of the system by investigating the quadrupole modes excited by the operator $Q$. An important case is the so called scissors mode. This mode corresponds to the rotation of a deformed condensate whose inclination angle $\theta$ oscillates in time. The oscillation can be induced by a sudden rotation of the confining trap with respect to 
the initial equilibrium configuration. It is important to point out that the frequency of this oscillation does not vanish with $\epsilon$ as one would expect for a classical system. In fact in a superfluid both the restoring force parameter and the mass parameter (moment of inertia) behave like $\epsilon^{2}$ so that the frequency keeps a constant value when $\epsilon \rightarrow 0$. The hydrodynamic theory of superfluids predicts the value $[18$

$$
\omega_{H D}=\sqrt{\omega_{x}^{2}+\omega_{y}^{2}}
$$

in excellent agreement with the experimental results recently carried out at Oxford [19] (see fig.5). Conversely in a classical gas one predicts the value $\omega=\left|\omega_{x} \pm \omega_{y}\right|$. Also these values have been tested experimentally with high accuracy by exciting the rotation of the gas at high temperatures, well above $T_{c}$.

\section{K. Quantized vortices}

Quantized vortices are one of the most spectacular manifestations of superfluidity (we will not discuss here other important manifestations of superfluidity, like the reduction of viscosity recently explored at MIT [20]). The existence of quantized vortices is the combined consequence of the behaviour of the phase of the order parameter, which fixes the irrotationality of the velocity field, and of the non-linearity of the equations of motion which is a crucial the consequence of two-body interactions. In the Gross-Pitaevskii theory a quantized vortex can be regarded as a stationary solution of eq.(13) of the form

$$
\Psi_{0}(\mathbf{r}, t)=e^{-i \mu t} e^{i \phi} \Psi_{v}(\mathbf{r})
$$

where $\phi$ is the azimuthal angle and $\Psi_{v}$ is a real function obeying the equation

$$
\left[-\frac{\hbar^{2} \nabla^{2}}{2 m}+\frac{\hbar^{2}}{2 m r_{\perp}^{2}}+\frac{m}{2}\left(\omega_{\perp}^{2} r_{\perp}^{2}+\omega_{z}^{2} z^{2}\right)+g \Psi_{v}^{2}\left(r_{\perp}, z\right)\right] \Psi_{v}\left(r_{\perp}, z\right)=\mu \Psi_{v}\left(r_{\perp}, z\right) .
$$

The velocity field associated with the order parameter (39) takes the form $\mathbf{v}=(\hbar / m) \nabla \phi=\hat{\mathbf{\Omega}} \times \mathbf{r} / r^{2}$ where $\hat{\boldsymbol{\Omega}}$ is the unit vector along the $z$-th direction. It satisfies the irrotationality constraint everywhere except along the vortical line and gives rise to a total angular momentum given by

$$
L_{z}=N \hbar \text {. }
$$

The "centrifugal" term in $1 / r_{\perp}^{2}$ of eq.(40) originates from the peculiar behaviour of the velocity field of the vortical configuration. This term is responsible for the vanishing of the condensate density $\left|\Psi_{v}\right|^{2}$ along the $z$-th axis and produces the so called vortex core, whose size is fixed by the healing length. In fig.6 we plot a typical vortical profile obtained by solving the Gross-Pitaevskii equation (40).

Quantized vortices are excited states of the system but they should not be confused with the elementary excitations discussed in sect.I . From the many-body point of view the vortex correspond to a state where all the N particles of the sample occupy the new solution of the GP equation. The excitation energy and the angular momentum associated with the vortex are consequently "macroscopic" quantities while the excitation energy and the angular momentum of elementary excitations are of the order, respectively, of the trapping frequencies and of a few units of $\hbar$.

The excitation energy $E_{v}$ of the vortex, given by the difference between the vortex and the ground state energies, can be calculated starting from the solution of the GP equation (40) and using expression (17) for the energy. For large N samples the calculation can be done analytically. One finds [21]

$$
E_{v}=\frac{4 \pi}{3} n_{0} Z \frac{\hbar^{2}}{m} \ln \frac{0.67 R_{\perp}}{\xi}
$$

where $n_{0}$ is the value of the density in the center of the trap. It is worth noticing that the factor in front of the logarithm is proportional to the so called column density $\int d z n(x, y, z)=(4 / 3) Z n_{0}$ calculated along the symmetry axis $(x=y=0)$. As we will see later this identification is useful for the calculation of the the energy of a vortex line displaced from the symmetry axis. Result (42) allows for an estimate of the critical angular velocity needed to generate an energetically stable vortex. In fact, calculating the energy $H-\Omega L_{z}$ of the sample in the frame rotating with angular velocity $\Omega$, one easily finds that the vortex is the lowest energy configuration if the angular velocity $\Omega$ exceeds the critical value 


$$
\Omega_{c r}=\frac{E_{v}}{L_{z}}=\frac{5}{2} \frac{\hbar}{m R_{\perp}^{2}} \ln \frac{0.67 R_{\perp}}{\xi} .
$$

In deriving result (43) we have used the relationship $N=(8 / 15) \pi n_{0} R_{\perp}^{2} Z$. Typical values of $\Omega_{c r}$ correspond to fractions of $\Omega_{\perp}$, the exact value depending on the geometry of the trap and on the value of the Thomas-Fermi parameter. In the recent experiments carried out at ENS-Paris [22] with an enolangated trap $\left(\omega_{z} / \omega_{\perp} \sim 1 / 10\right)$ the calculated value of $\Omega_{c r}$ turns out to be about $\omega_{\perp} / 3$. In this experiment the vortex is created by rotating a slightly asymmetric trap at different values $\Omega$, in analogy with the rotating bucket experiment on superfluid helium. This procedure differes from the one followed at JILA [25] where the vortex was instead created with full optical methods. The value of the critical angular velocity measured at ENS is a factor 2 higher than the theoretical estimate (43). The discrepancy is due to the fact that, even if the vortex is energetically favourable, its nucleation is forbiddeen by the occurrence of a barrier which disappears only at higher angular velocities. The nucleation of the vortex as well as the study of its stability and life time is at present a topic of intense experimental and theoretical investigation.

An important question in the study of vortices is not only how to produce but also how to detect them. The size of the vortex core is in fact too small (fractions of microns) to be observed in situ. It can be however observed by imaging the atomic cloud after expansion. An alternative method is given by the measurement of angular momentum, a quantity which, according to eq.(41), characterizes in a peculiar way the quantum nature of the vortex. The presence of angular momentum produces a splitting of the quadrupole frequencies $\omega_{ \pm}$relative to the excitations with $\ell_{z}= \pm 2$ ( $\ell_{z}$ is the third component of the agular momentum of the elementary excitation). The value of the splitting and its relation with the angular momentum $L_{z}$ of the vortex can be derived using a sum rule approach. The result, in the Thomas-Fermi limit, is given by the formula [23]

$$
\omega_{+}-\omega_{-}=2 \frac{<L_{z}>}{m<r_{\perp}^{2}>} .
$$

The splitting of the quadrupole frequencies $\omega_{ \pm}$produces a mechanism of precession that can be detected experimentally. To this purpose one generates a small quadrupole deformation in the plane orthogonal to the vortex axis, by adding a field of the form $x^{2}-y^{2}$. The precession of the quadrupole deformation is directly related to the splitting (44). In fact, by requiring that in the frame rotating with angular velocity $\Omega_{\text {prec }}$ the energies $\omega_{ \pm}$of the two modes are degenerate, one finds the result $\Omega_{\text {prec }}=\left(\omega_{+}-\omega_{-}\right) / 4$. Using the above procedure the authors of ref [24] have observed a jump in the angular momentum of the condensate above a critical value of the stirring frequency (see fig. 7). This approach has permitted to test the quantization of the angular momentum of a single vortex line.

Gyroscopic effects of different type have been observed in [25] where the precession of a vortex line circulating around the symmetry axis of the condensate was investigated. The angular velocity characterizing this precession can be estimated by evaluating the energy and the angular momentum of a vortex line as a function of its distance $d$ from the symmetry axis. This calculation is easy if one assumes that the vortex line is straight. The $d$ dependence of the energy is determined, within logarithmic accuracy, by the $d$-dependence of the column density $\int d \mathbf{r} n(x, y, z)$ characterizing the prefactor of eq.(42). This yields [?]

$$
E_{v}=N \hbar \Omega_{c}\left(1-\frac{d^{2}}{R_{\perp}^{2}}\right)^{3 / 2}
$$

where $\Omega_{c}$ is the critical angular velocity (43). Using Stokes' theorem and working in the Thomas-Fermi limit it is possible to calculate also the angular momentum for which one finds the result

$$
L_{z}=N \hbar\left(1-\frac{d^{2}}{R_{\perp}^{2}}\right)^{5 / 2} .
$$

The angular velocity characterizing the precession is given by $\Omega_{p r e c}=\partial E_{v} / \partial L_{z}$. For small values of the vortex displacement $\left(d \ll R_{\perp}\right)$ one obtains the result [26] $\Omega_{\text {prec }}=(3 / 5) \Omega_{c r}$ which agrees reasonably well with the experimental results of JILA [27].

\section{Conclusions and acknowledgements}

The main purpose of these notes was to give an introduction to some key features which have been the object of recent experimental and theoretical work in the physics of trapped Bose-Einstein condensates. Special emphasis was given to the interplay between the phase of the order parameter and two-body interactions which characterize the 
non linearity of the Gross-Pitaevskii equation. This interplay is at the basis of the superfluid behaviour exhibited by these systems and is responsible, in particular, for the existence of quantized vortices.

It is a pleasure to thank the fruitful collaboration of the BEC Trento team and the warm hospitality of the French friends at the Institute d'Etudes Sientifiques de Cargèse.

[1] F. Dalfovo et al., Rev. Mod. Phys. 71, 463 (1999).

[2] Ensher et al., Phys. Rev. Lett. 77, 4984 (1996).

[3] Mewes et al., Phys. Rev. Lett. 77, 416 (1996).

[4] G. Baym. et al. Phys. Rev. Lett. 83, 1703 (1999).

[5] S. Giorgini, L. Pitaevskii and S. Stringari, Phys. Rev A 54, 4633 (1996).

[6] P. Sokol, in Bose-Einstein Condensation, edited by A. Griffin et al. (Cambridge University Press, 1995$)$ p.51.

[7] O. Penrose and L. Onsager, Phys. Rev. 104, 576 (1956).

[8] D.S. Lewart, V.R. Pandharipande and S.C. Pieper, Phys. Rev. B 37, 4950 (1988).

[9] E.P. Gross, nuovo Cimento 20, 454 (1961); L.P. Pitaevski, Zh. Eksp. Teor. Fiz. 40, 646 (1961) [Sov. Phys. JETP 13, 451 (1961).

[10] W. Ketterle, D.S. Durfee and D.M. Stamper-Kurn, in Proceedings of the International School of Physics "E. Fermi", Course CXL, M. Inguscio, S. Stringari and C. Wieman (Eds.), IOS Press, Amsterdam 1999, p.67.

[11] J. Stenger et al., Phys. Rev. Lett. 83, 2876 (1999).

[12] M.R. Andrews et al., Science 275, 637 (1997).

[13] S. Stringari, Phys. Rev. Lett. 77, 2360 (1996).

[14] M.R. Andrews et al., Phys. Rev. Lett. 79553 (1997).

[15] E. Zaremba, Phys. Rev. A, 57, 518 (1998); S. Stringari, Phys. Rev. A, 58, 2385 (1998).

[16] D.M. Stamper-Kurn et al., Phys. Rev. Lett. 81, 500 (1998).

[17] G.B. Hess and W.M. Fairbank, Phys. Rev. Lett. 19, 216 (1967).

[18] D. Guery-Odelin and S. Stringari, Phys. Rev. Lett. 83, 4452 (1999).

[19] O.M. Marago' et al., Phys. Rev. Lett. 842056 (2000).

[20] C. Raman et al., Phys. Rev. Lett. 83, 2502 (1999); A.P. Chikkatur et al., Phys. Rev. Lett. 85, 483 (2000).

[21] E. Lundh, C.J. Pethick and H. Smith, Phys. Rev. A 55, 2126 (1997).

[22] K.W. Madison et al., Phys. Rev. Lett. 84, 806 (2000).

[23] F. Zambelli and S. Stringari, Phys. Rev. Lett. 81, 1754 (1998).

[24] F. Chevy, K.W. Madison and J. Dalibard, Phys. Rev. Lett. 85, 2223 (2000).

[25] M.R. Matthews et al., Phys. Rev. Lett. 83, 2498 (1999).

[26] A. Svidzinsky and A. Fetter, J. Phys. G, in press, and references therein.

[27] B.P. Anderson et al., Phys. Rev. Lett. 85, 2857 (2000).

\section{FIGURE CAPTION:}

Fig.1. Condensate fraction of a trapped Bose gas as a function of $T / T_{c}$. Circles are the experimental results of [2, while the dashed line is eq.(俚).

Fig.2. Condensate fraction as a function of temperature in superfluid ${ }^{4} \mathrm{He}$ (from [6]).

Fig.3. Total density and condensate density profiles of a helium droplet of 70 atoms (from [8]).

Fig.4. Axial profiles of a cloud of sodium atoms at different temperatures (from $[10]$ ).

Fig.5. Scissors oscillation observed in [19]. The full line corresponds to a sinusoidal fit with $\omega / 2 \pi=265 \pm 0.8 \mathrm{~Hz}$ to be compared with the theoretical prediction $265 \mathrm{~Hz}$ of eq.38.

Fig.6. Density profile of a quantized vortex (from [1]).

Fig.7. Angular momentum per particle of a trapped condensate as a function of the angular velocity of the trap (from [24]). 


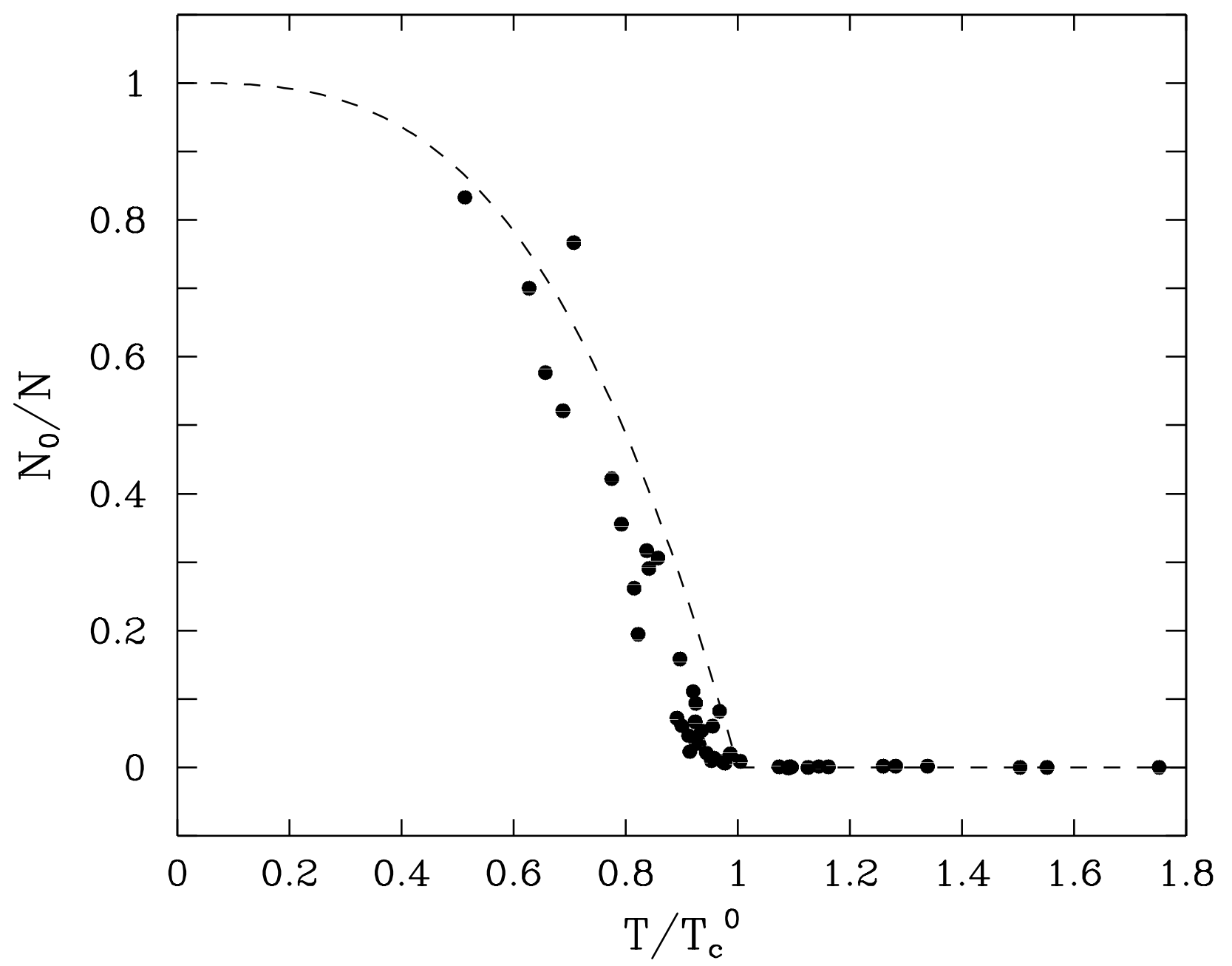




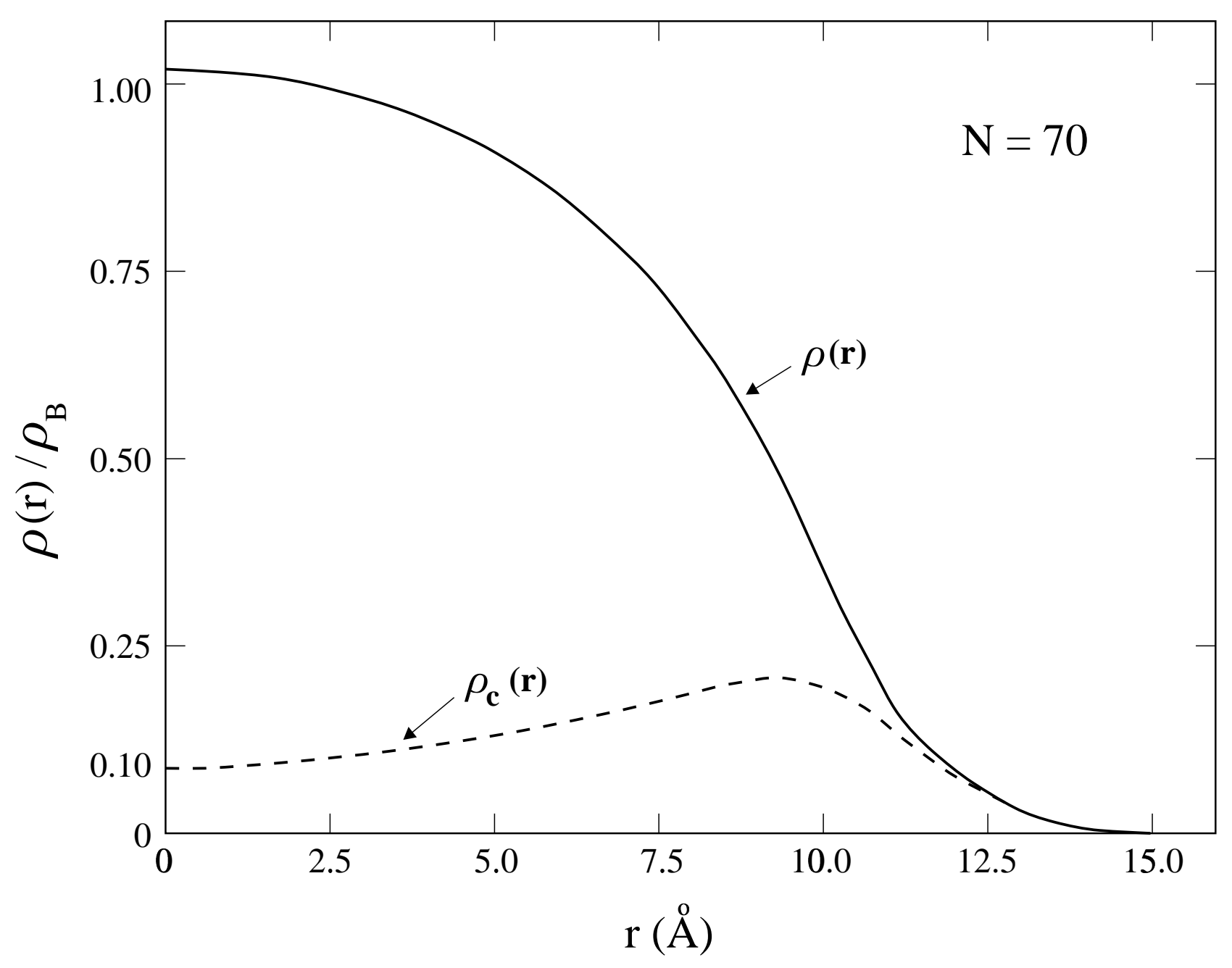




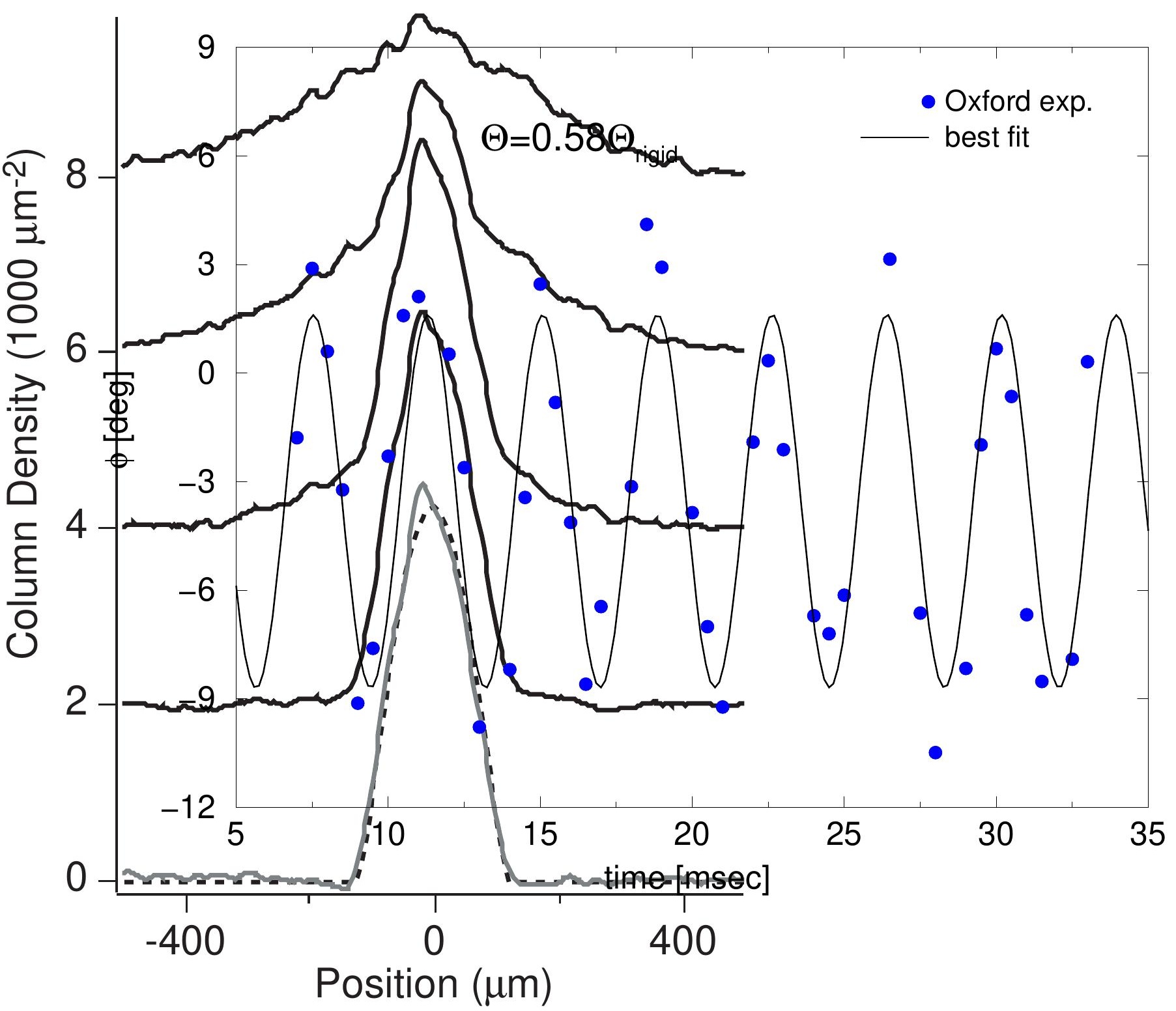




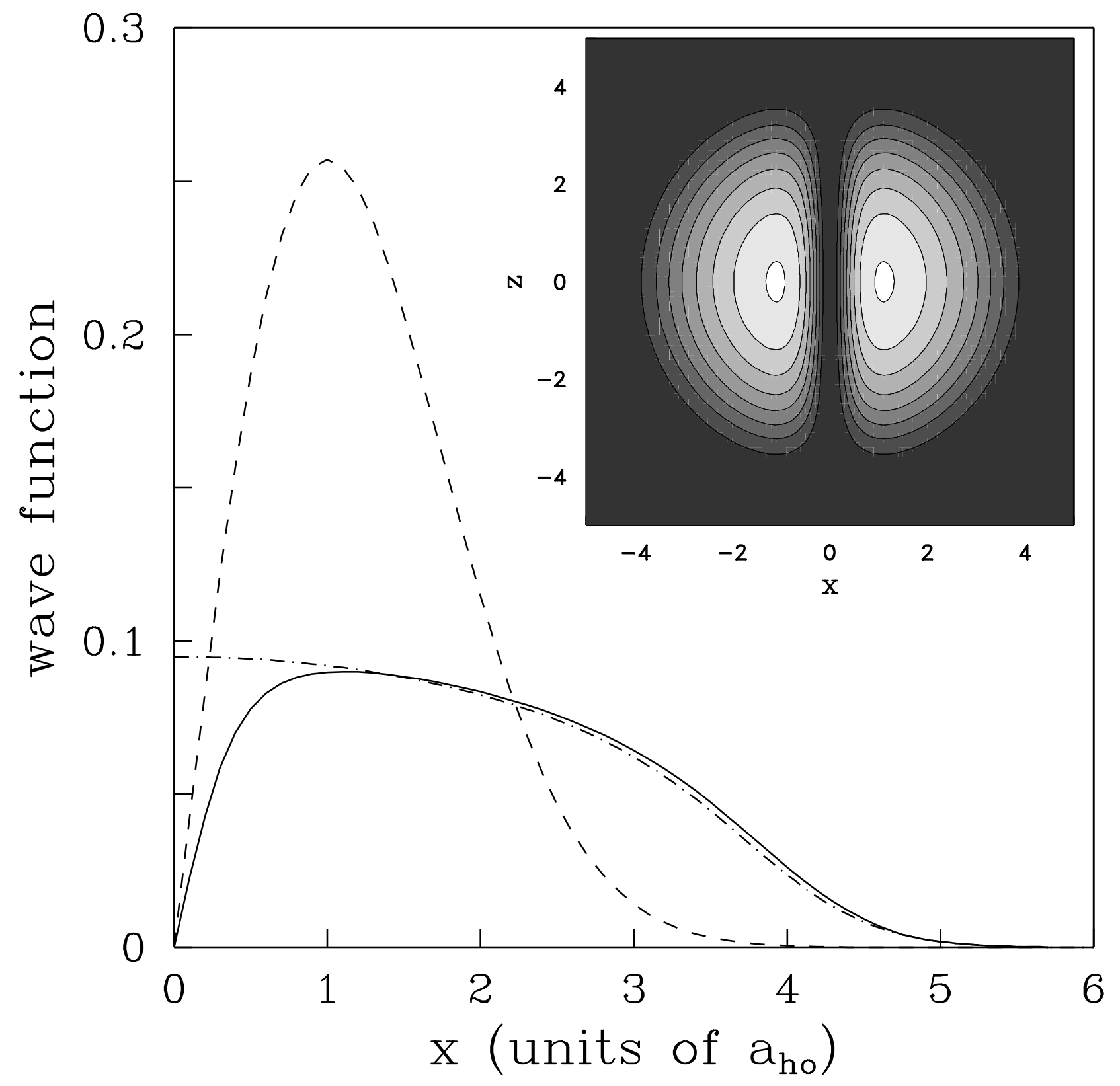




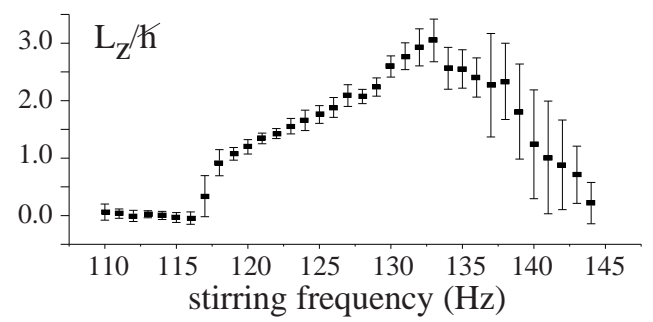

